\title{
Ethylenediamine modified rice hull as a sorbent for the removal of Basic Blue 3 and Reactive Orange 16
}

\begin{abstract}
The potential of ethylenediamine modified rice hull to remove both basic and reactive dyes from aqueous solutions was studied. Equilibrium sorption data could be fitted into Langmuir and Brunauer-Emmett-Teller isotherm models. Sorption was enhanced by 4.5- and 2.4-fold for Basic Blue 3 and Reactive Orange 16, respectively, in binary dye solutions compared to single dye solutions. Column studies revealed that breakthrough was bed-depth, flow-rate and influent-concentration dependent. Unusual breakthrough curves were obtained for Reactive Orange 16, with very rapid initial breakthrough followed by complete retention at low flow rate, influent concentration and high bed depth. The breakthrough curves of Basic Blue 3 followed the typical $S$ shape of packed-bed systems. Theoretical breakthrough curves at different bed depths and flow rates generated by the two-parameter mathematical model agreed well with the experimental data of single dye solution of Basic Blue 3.
\end{abstract}

Keyword: sorption, rice hull, ethylenediamine, Reactive Orange 16, Basic Blue 3, twoparameter model, dye removal, wastewater treatment, reactive dyes, basic dyes, bed depths, flow rates, mathematical modelling, biosorption 